\title{
A THEOREM OF C. RYLL-NARDZEWSKI AND METRIZABLE L.C.A. GROUPS
}

\author{
L. THOMAS RAMSEY
}

\begin{abstract}
Gamma$ denotes a metrizable locally compact abelian group and $\bar{\Gamma}$ its Bohr compactification. Let $\gamma \in \Gamma$ be a cluster point of some subset $E$ of $\Gamma$ in the topology of $\Gamma$. Then there are two disjoint subsets of $E$ which also cluster at $\gamma$ in the Bohr group topology. The proof is elementary and provides a new proof of the theorem of C. Ryll-Nardzewski on cluster points of $I$-sets in $R$. Given the continuum hypothesis, either theorem characterizes metrizability in locally compact abelian groups. One of these characterizations is shown to be equivalent to the continuum hypothesis.
\end{abstract}

$\Gamma$ denotes a locally compact abelian group whose dual is $G . \bar{\Gamma}$, the Bohr compactification of $\Gamma$, is the 1.c.a. group that is dual to $G_{d}, G$ with the discrete topology.

THEOREM 1. Suppose that $\Gamma$ is metrizable. Let $E$ be a subset of $\Gamma$ which clusters at $\gamma \in \Gamma$ in the topology of $\bar{\Gamma}$. Then there are two disjoint subsets of $E$ which likewise cluster at $\gamma$.

Proof. A basic neighborhood $U=U\left(\gamma ; g_{1}, \ldots, g_{n} ; \varepsilon\right)$ of $\gamma$ in $\bar{\Gamma}$ is determined by a finite set of points $g_{1}, \ldots, g_{n}$ from $G$ and some $\varepsilon>0$. Precisely, $U$ consists of $\lambda$ in $\bar{\Gamma}$ such that $\left|\lambda\left(g_{i}\right)-\gamma\left(g_{i}\right)\right|<\varepsilon$ for $1<i<n$. Because $\gamma$ is a cluster point of $E$ in $\bar{\Gamma}$, given any finite subset $F \subseteq E$ there is some $\lambda \in E \backslash F$ such that $\lambda \in U$. Since both $\lambda$ and $\gamma$ are from $\Gamma$ and thus continuous on $G$ with respect to the original topology, there is some neighborhood $V$ of $\left(g_{1}, \ldots, g_{n}\right)$ in $G^{n}$ such that $\left(h_{1}, \ldots, h_{n}\right) \in V$ implies $\lambda \in U\left(\gamma ; h_{1}, \ldots, h_{n} ; 3 \varepsilon\right)$. For any compact subset $K$ of $G, K^{n}$ is covered by a finite number of such $V$ 's. We may conclude that for $K$ a compact subset of $G$, for $n>0$, for $\varepsilon>0$, and $F$ a finite subset of $E$, there exist $\lambda_{1}, \ldots, \lambda_{m}$ in $E \backslash F$ such that $\left(h_{1}, \ldots, h_{n}\right) \in K^{n}$ implies that for some $1<j<m$, $\lambda_{j} \in U\left(\gamma ; h_{1}, \ldots, h_{n} ; \varepsilon\right)$. Denote the set $\left\{\lambda_{1}, \ldots, \lambda_{m}\right\}$ as $D(K, n, \varepsilon, F)$.

We now use the hypothesis that $\Gamma$ is metrizable. This occurs exactly when $G$ is $\sigma$-compact. Let $G$ be $\cup_{n=1}^{\infty} K_{n}$ with each $K_{n}$ compact and $K_{n} \subseteq K_{n+1}$. Define two sequences of finite subsets of $E$ inductively. Set $T_{0}=S_{0}=\varnothing$. Let $S_{n+1}=$ $D\left(K_{n+1}, n+1,(n+1)^{-1}, F_{n+1}\right)$ where $F_{n+1}=\cup_{j<n}\left(T_{j} \cup S_{j}\right)$. Then $T_{n+1}=$ $D\left(K_{n+1}, n+1,(n+1)^{-1}, F_{n+1} \cup S_{n+1}\right)$. Clearly $\cup_{n} T_{n}$ and $\cup_{n} S_{n}$ are disjoint subsets of $E$ which cluster at $\gamma$ in $\bar{\Gamma}$.

Received by the editor November 29, 1978.

AMS (MOS) subject classifications (1970). Primary 43A25, 43A60; Secondary 03E50.

$K e y$ words and phrases. Bohr compactification, l.c.a. groups, $I$-sets, continuum hypothesis. 
Definition. A subset $E$ of $\Gamma$ is said to be an $I$-set if every bounded function on $E$ can be extended to $\bar{\Gamma}$ as a continuous function. Equivalently, $E \subset \Gamma$ is an $I$-set if every bounded function on $E$ is the restriction to $E$ of an almost periodic function on $\Gamma[1$, p. 32].

The following result due to C. Ryll-Nardzewski [2] for $\Gamma=R$ is a corollary of Theorem 1.

COROllary. Let $\Gamma$ be a metrizable l.c.a. group. If $E \subset \Gamma$ is an I-set, then $E$ has no points of $\Gamma$ as cluster points in $\bar{\Gamma}$. Consequently the union of $E$ with any finite subset of $\Gamma$ is also an I-set.

The theorem and the corollary are both false when $G=T_{d}$, the circle with the discrete topology. In that case $\Gamma=\bar{\Gamma}=\bar{Z}$, the Bohr compactification of the integer group $Z$. The set $E$ which consists of powers of 3 is an $I$-set, both as a subset of $Z$ and of $\Gamma$. The set $E$ has uncountably many cluster points $(\Gamma=\bar{\Gamma})$. This example is characteristic of nonmetrizable groups if the continuum hypothesis is assumed; i.e., $2^{k_{0}}=x_{1}$. In what follows $(\mathrm{CH})$ indicates use of the continuum hypothesis.

Proposition (CH). Let $\Gamma$ be a nondegenerate compact abelian group, and let $I$ be an uncountable set. Then there is an embedding of the Stone-Cech compactification $\beta(\mathbf{N})$ in the group $\Gamma^{I}$, where $\mathbf{N}$ is the set of natural numbers.

Proof. Let 2 be the discrete two-point space. Since $\mathbf{N}$ is discrete, the evaluation map ev: $\mathbf{N} \rightarrow 2^{2^{N}}$ extends to an embedding of $\beta(\mathbf{N})$ into $2^{2^{N}}$. Since $I$ is uncountable, there is an injection of $2^{\mathrm{N}}$ into $I$, and this then allows an embedding of $2^{2^{\mathrm{N}}}$ into $\Gamma^{I}$, since $\Gamma$ is nondegenerate and compact. The composition of the embedding of $\beta(N)$ into $2^{2^{N}}$ with this embedding into $\Gamma^{I}$ then yields the desired embedding.

Lemma 1. Let $\Gamma$ and $\Gamma^{\prime}$ be locally compact abelian groups, and let $f: \Gamma \rightarrow \Gamma^{\prime}$ be a continuous surmorphism of $\Gamma$ onto $\Gamma^{\prime}$. Then any I-set in $\Gamma^{\prime}$ lifts to an I-set in $\Gamma$.

Proof. Let $E$ be an $I$-set in $\Gamma^{\prime}$. For each point of $E$, choose a point in $\Gamma$ which maps onto it under $f$, and call the resulting set $F$. Then $f \mid F: F \rightarrow E$ is a bijection. Since $f$ is a continuous surmorphism, $f$ extends to a continuous surmorphism $\bar{f}$ : $\bar{\Gamma} \rightarrow \bar{\Gamma}^{\prime}$.

Now, let $g: F \rightarrow \mathrm{C}$ be any bounded function. Then $g \cdot f^{-1}$ is a bounded function on the $I$-set $E$, and so this extends to a continuous function $g_{1}: \bar{\Gamma}^{\prime} \rightarrow C$. The composition $g_{1} \cdot \bar{f}: \bar{\Gamma} \rightarrow \mathbf{C}$ then provides the desired extension of $g$ to $\bar{\Gamma}$. Thus $F$ is an $I$-set in $\Gamma$.

Below ${ }^{I} H$ and $H^{I}$ denote direct sums and direct products of the group $H$, respectively.

LEMMA 2. Let $G$ be an uncountable abelian group. Then $G$ has a subgroup $G^{\prime}$ which is isomorphic to ${ }^{I} H$, where $H$ is a nondegenerate group, and $I$ is an uncountable set (of the same cardinality as $G$ ). 
Proof. Let $D$ be the divisible hull of $G$, i.e., $D$ is aminimal divisible extension of $G$. Then $D$ can be represented as ${ }^{\left(r_{0}\right)} \mathbf{Q} \oplus \oplus_{p}^{\left(r_{p}\right)} Z\left(p^{\infty}\right)$ where $\mathbf{Q}$ is the group of rationals, $r_{0}$ is the torsion-free rank of $G$, and $r_{p}$ is the $p$-rank of $G$ for each prime $p$ in $P$, the set of primes [3, Appendix A, pp. 444-446]. Now, $D$ is uncountable since $G$ is (in fact of equal cardinality), and so either $r_{0}$ is uncountable, or $r_{p}$ is uncountable for some prime $p$. In any event, we conclude that $G$ has an uncountable set of independent elements all having the same order. The group $G^{\prime}$ that this set generates is then isomorphic to ${ }^{I} H$, where $H$ is the cyclic group of the order in question, and $I$ is the set of independent elements of the order.

Theorem 2 below is a converse of Theorem 1 under the continuum hypothesis. Theorems 1 and 2 together characterize metrizable locally compact abelian groups under the continuum hypothesis assumption.

TheOREM $2(\mathrm{CH})$. Let $\Gamma$ be a nonmetrizable l.c.a. group. Then there is an infinite $I$-set $E \subset \Gamma$ whose closure in $\Gamma$ is a subset of $\Gamma$. Consequently, Theorem 1 and its corollary are false for $\Gamma$.

Proof. Suppose that $\Gamma$ is a nonmetrizable locally compact abelian group. Then, according to the Principle Structure Theorem for locally compact abelian groups $[1$, p. 40], $\Gamma$ has an open subgroup which is a direct product of a vector group $V$ and a compact subgroup $\Gamma^{\prime}$ of $\Gamma$. Since $V$ is metrizable and $\Gamma$ is not, and since $V \times \Gamma^{\prime}$ is open, it follows that $\Gamma^{\prime}$ is also not metrizable. Now, Tietze's Extension Theorem implies that any $I$-set in $\Gamma^{\prime}$ is also an $I$-set in $\Gamma$. Moreover, since $\Gamma^{\prime}$ is compact and the map from $\Gamma$ to $\bar{\Gamma}$ is continuous, the image of $\Gamma^{\prime}$ in $\bar{\Gamma}$ is closed, and so any $I$-set in $\Gamma^{\prime}$ has its closure a subset of $\Gamma^{\prime}$, and hence of the image of $\Gamma$ in $\bar{\Gamma}$. This shows that it suffices to produce the desired $I$-set in $\Gamma^{\prime}$, or said another way, it suffices to consider the case when $\Gamma$ is compact.

Given that $\Gamma$ is a compact nonmetrizable abelian group, its character group $G$ is then an uncountable abelian group. Thus, Lemma 2 implies that $G$ has a subgroup $G^{\prime} \simeq^{I} H$, where $H$ is a nondegenerate group, and $I$ is uncountable. Then, the character group $\Gamma^{\prime}$ of $G^{\prime}$ is a quotient of $\Gamma$, and is isomorphic to $\Delta^{I}$, where $\Delta$ is the character group of $H$. Lemma 1 implies that it suffices to produce the desired $I$-set in $\Gamma^{\prime}$. Moreover, the proposition implies that $\beta(\mathbf{N})$ is embedded in $\Gamma^{\prime}$, and this clearly makes the corresponding copy of $\mathbf{N}$ an $I$-set in $\Gamma^{\prime}$, and we are done.

The existence of an infinite $I$-set in an arbitrary nonmetrizable l.c.a. group is actually equivalent to the continuum hypothesis. To see this, let $G$ be a discrete group whose cardinality is the first uncountable cardinal and let $E$ be an infinite $I$-set in $G^{\wedge}=\Gamma$. For each partition of the set $E$ into two disjoint subsets, choose a finitely-supported discrete measure with rational coefficients whose FourierStieltjes transform is approximately 1 on one subset of the partition of $E$ and approximately 0 on the other subset of $E$. The cardinality of the set of such discrete measures is the same as that of $G$ but the cardinality of possible partitions of $E$ is at least $2^{\aleph_{0}}$. Thus, $2^{\aleph_{0}}<\aleph_{1}$, i.e. the continuum hypothesis holds.

It is also false (without $\mathbf{C H}$ ) that the theorem of $\mathrm{C}$. Ryll-Nardzewski is characteristic of metrizable l.c.a. groups. This follows from the preceding observation. 
Otherwise we could have a compact, nonmetrizable group in which every $I$-set were finite and hence the Ryll-Nardzewski theorem would hold for that nonmetrizable group.

The author wishes to thank the referee for his reading of the original manuscript. The referee shortened and greatly clarified the proof of Theorem 2 . He improved Lemma 2 by noting the reference to [3]. This allowed him to conclude that an uncountable direct sum of a single group $H$ occurred as a subgroup of $G$.

\section{REFERENCES}

1. Walter Rudin, Fourier analysis on groups, Wiley, New York, 1969.

2. C. Ryll-Nardzewski, Concerning almost periodic extensions of functions, Colloq. Math. 12 (1964), 235-237.

3. Edwin Hewitt and Kenneth A. Ross, Abstract harmonic anaylsis, Academic Press, New York, 1963.

Departmient of Mathematics, University of HawaIl, Honolulu, HawaI 96822 